\title{
Toward an SDN-based Data Collection Scheme for Vehicular Fog Computing
}

\author{
Abdelwahab Boualouache, Ridha Soua, and Thomas Engel \\ SnT, University of Luxembourg, Luxembourg \\ Email: \{abdelwahab.boualouache, ridha.soua, thomas.engel\}@uni.lu
}

\begin{abstract}
With the integration of fog networks and vehicular networks, Vehicular Fog Computing (VFC) is a promising paradigm to efficiently collect data for improving safety, mobility, and driver experience during journeys. To this end, we exploit the Software-Defined Networking (SDN) paradigm to propose a fully-programmable, self-configurable, and context-aware data collection scheme for VFC. This scheme leverages a stochastic model to dynamically estimate the number of fog stations to be deployed. Our simulation results demonstrate that our proposed scheme provides lower latency and higher resiliency compared to classical data collection schemes.
\end{abstract}

Index Terms-Vehicular networks; Fog computing; Vehicular Fog Computing; Data Collection; Software Defined Networking.

\section{INTRODUCTION}

The safety and mobility of connected vehicles are expected to be improved through enhanced situational awareness and prediction [1]. The cornerstone of these two future developments is data that is being collected by vehicles, recorded and exchanged through vehicle-to-vehicle (V2V) and vehicleto-infrastructure (V2I) communications. Additionally, in the recent years, cloud computing has emerged as a major trend in information technology. Indeed, many vehicular cloudbased solutions [2] came out given the vehicles' increasing need for more computing, storage, and processing resources. Vehicular Cloud Computing (VCC) takes advantage of cloud computing to serve vehicles' drivers and allows a large amount of content to be easily processed by distant cloud services [3]. Several vehicular applications collect data from proximitybased resources to provide various real-time services for their users. However, this has the downside of resulting in explicit latency for the service, this being especially problematic when the application requires a fast response in the edge network. This shows the benefits of bringing fog computing to vehicular networks. Indeed, the fusion of fog computing and vehicular networks has led to the emergence of Vehicular Fog Computing networks (VFC) [4]. VFC not only deploys highly virtualized computing and communication services near vehicles, but also distributes data processing tasks to fog nodes located in the vicinity of the vehicles producing the data and the end-users requesting it in order to reduce the latency $[5,6]$. Nevertheless, despite the recent integration of fog computing into vehicular networks, data collection has not been well investigated in VFC. To the best of our knowledge, only [7] and [8] have tackled this issue. However, their proposed approaches to exploit the benefits of VFC and do not take into consideration data dissemination and the deployment cost of fog stations.

In this paper, we propose an efficient SDN-based data collection method for VFC. The proposed scheme is fully programmable, self-configurable, and context-aware thanks to the SDN control plane, which is responsible for: (i) configuring data collection parameters, (ii) deployment of fog stations, and (iii) data routing from vehicles to fog stations. The main contributions of this paper can thus be summarized as follows:

1) A novel SDN-based scheme is proposed to efficiently collect data in VFC.

2) An accurate stochastic model to estimate the number of fog stations to deploy according to vehicular traffic density and availability of data. This model is validated using realistic road traffic measurements from Luxembourg city.

3) A performance evaluation, through extensive simulations, of the proposed scheme in terms of latency and resiliency compared to classical data collection schemes.

The remainder of this paper is organized as follows. Section II describes the related work. The system model considered is presented in section III. The proposed SDN-based data collection scheme is described in Section IV. Section V presents the stochastic model to estimate the number of fog stations to be deployed. The performance evaluation results are given in Section VI. Finally, Section VII concludes the paper.

\section{RELATED WORK}

Many data collection schemes have been proposed for vehicular networks [9]. Turcanu et al. [10] proposed a protocol for collecting and disseminating data among vehicles over a large vehicular area. The collected data is disseminated in a multi-hop manner to a single RSU using the IEEE $802.11 \mathrm{p}$ communication standard. The proposed protocol is adaptive to different levels of vehicular traffic density. The authors of [11] carried out a simulation study of proactive and reactive data collection schemes. Obtained results show that proactive schemes consume less bandwidth and have lower latency compared to reactive schemes. Consequently, the authors propose a proactive data collection protocol which uses only $3 \mathrm{G} / \mathrm{LTE}$ communications and adapts some polling period variables to provide accurate traffic data. Jiao et al. [12] 
proposed a data collection scheme which relies on both IEEE 802.11p and cellular communications. The IEEE 802.11p protocol is mainly used to disseminate collected data to Road Side Units (RSUs). The software-defined networking (SDN) paradigm is used to establish the dissemination path from vehicles to RSUs. Cellular communications are used only when the dissemination path cannot be established or when collected data is urgent.

In all the aforementioned works, the collected data is sent to a distant monitoring center (cloud). Vehicles then need to request the data from the cloud, which may result in undesirably long response times and prevent vehicles from taking advantage of the readily-available data. By deploying fog stations in the proximity of vehicles, VFC is an effective way to overcome this issue. Lai et al. [7, 8] propose a data collection scheme for VFC. The authors propose deploying a fog station at every RSU. Vehicles continuously calculate the weight of each sensed data item. This weight is computed based on the correlation between the data, the event and the confidence in the sensed data. If this weight is greater than a given threshold, the vehicle sends this observation to the nearest RSU. Each RSU collects these one-hop received observations to calculate the global weight. If the global weight is greater than a second threshold, the RSU initiates the event-checking procedure wherein some vehicles are selected to switch to deep sensing mode and start collecting for dissemination them to fog stations. However, this scheme has several drawbacks: (i) long latency, because data collection is performed in a reactive manner and a significant time is needed for checking; (ii) absence of a mechanism to disseminate data to fog stations; (iii) a significant deployment cost for fog stations.

\section{SySTEM MODEL}

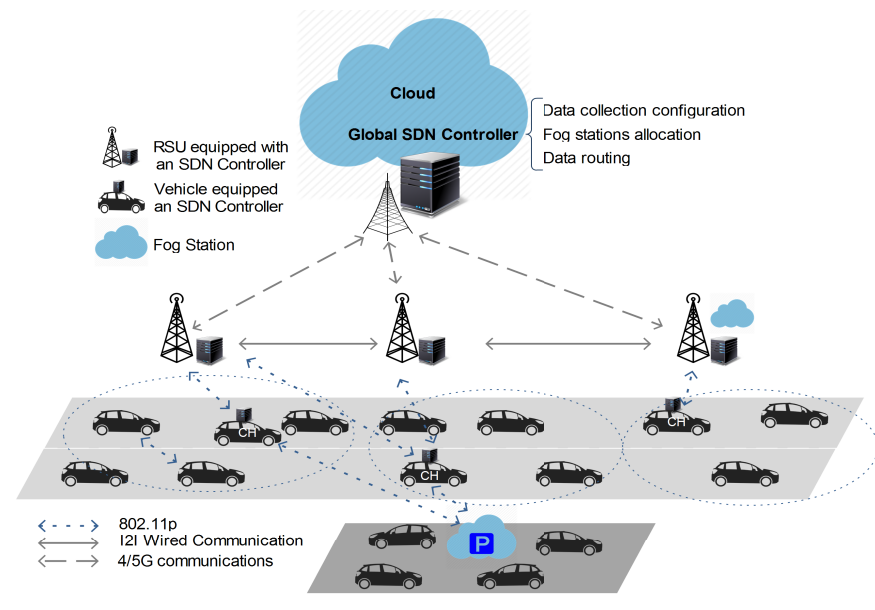

Fig. 1: Software-defined vehicular fog computing architecture

As illustrated in Figure 1, we consider a software-defined architecture for VFC that consists of three layers:

- the infrastructure layer: this includes vehicles equipped with sensors to collect data from the surrounding envi- ronment. Each vehicle is also equipped with an IEEE $802.11 \mathrm{p}$ interface to communicate with nearby vehicles and with RSUs in the vicinity. Vehicles periodically form clusters. Each cluster is managed by one Cluster Head $(\mathrm{CH})$, which is equipped with a local SDN controller. Each Cluster Member (CM) is also equipped with an SDN agent that allows interaction with the local SDN controller running at the $\mathrm{CH}$. The communication in this layer are multi-hop V2V. V2I communications are used to communicate with the other layers.

- the fog layer: this consists mainly of RSUs. Each RSU is equipped with two interfaces: (a) a wired link to communicate with the neighboring RSUs, and (b) a $4 \mathrm{G} / 5 \mathrm{G}$ interface to communicate with the global SDN controller. The RSUs are also equipped with regional SDN controllers and may also play the role of a fog node. This layer also includes vehicular fog stations, which are defined as a set of parked vehicles which collaborate and form a single fog station [2].

- the cloud layer: this layer is located on above of two previous layers. It includes the global SDN controller, which has global knowledge of such things as the mobility of vehicles and the density of the considered network. The global SDN controller is hosted in a distant location. The communication links between the local SDN controller and the vehicles are secured. We assume also that the communication links between the SDN controllers of the three levels of control are secured.

The control plane, which is made up of the global SDN controller, regional controllers (RSUs) and local controllers (CHs) is mainly responsible for (i) configuration of the data collection process, which includes initializing data collection parameters and their update - for more detail, Section IV; (ii) the deployment of fog stations, which consists of periodic estimation of the optimal number of fog stations to be deployed and their placement as near as possible to vehicles as described in V; and (iii) the data routing, which defines the routes that the data will follow from its origin (vehicles) to its destination (fog stations). Several existing SDN-based routing protocols can be used for this purpose [13].

\section{SDN-BASED DATA COLLECTION SCHEME FOR VEHICULAR FOG COMPUTING}

Here we detail our proposed SDN-based data collection scheme for VFC. As illustrated in Figure 2, this scheme consists of four phases: (i) initialization: during this phase, the global SDN controller initializes the Data Collection (DC) parameters; (ii) automatic data collection: in this phase, data is collected using the DC parameters and sent to fog stations; (iii) data request: where users request data by sending requests to deployed fog stations; and finally (iv) DC parameter update: in this phase, data collection parameters are updated and adapted according to the current context, such as the road traffic density and the demand for data. Phases (ii), (iii), and (iv) run in parallel for the whole system lifetime. 


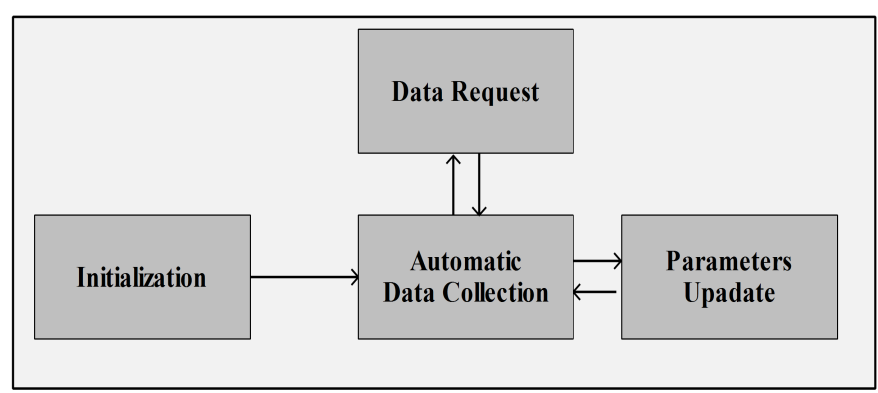

Fig. 2: The phases of SDN-based data collection scheme

\section{A. Initialization}

During this phase the default DC parameters are defined by the global SDN-controller and forwarded to the SDN controllers at different levels (regional and local). These parameters can be classified into two categories: (1) data-oriented parameters, and (2) fog-oriented parameters.

1) Data-oriented parameters: These include:

- Data Category: Our scheme allows system users to specify which data categories should be collected. For instance, a vehicular network generates various types of data that pours from a huge number of sensors. We differentiate: Road safety (collision alerts, congestion information, and road conditions); and Comfort data such as weather, free parking space, air pollution levels, etc.

- Data type: This parameter determines whether the data delivery has time constraints or not. Hence, we consider two types of data: (i) Delay Tolerant Data (DTD): which does not present any time constraints, and (ii) Real-Time Data (RTD): this data is considered time-sensitive and consequently requires to be delivered with a minimized latency.

- Importance factor: This parameter is associated with each data category. The purpose of introducing this parameter is to specify the quota of data to be collected for each category. In the initialization phase, the importance factors of all categories have the same value and are computed according to the following formulas: $F I_{i}=1 / N b_{c}$, where $F I_{i}$ is the importance factor of the category $i$ and $N b_{c}$ is the number of categories.

2) Fog-oriented parameters: These include:

- Deployment of fog stations: During the initialization phase, the global SDN controller specifies the default number of fog stations to use for data collection, and their storage capacity. It also chooses the RSUs that will be considered to be fog stations, and create some vehicular fog stations if necessary. Afterwards the number of fog stations will be periodically updated according to vehicular density and the availability of data.

- Assignment of fog stations: The vehicles that belong to the same cluster send their collected data to the same fog station. For this reason, each vehicular cluster should be assigned to one fog station. To this end, a default assignment of fog stations is performed during the initialization phase. This assignment is periodically updated according to the mobility pattern of vehicles.

After the definition of the default data collection parameters, the operation mode of our fog-assisted data collection scheme is based on three distinct steps. Firstly, the global SDN controller selects the set of RSUs that will host the fog stations and the vehicular fog stations to be created. Then, the global SDN controller assigns to each cluster a fog station to store its data. The assignment messages are forwarded from the global SDN controller to local SDN controllers through regional SDN-controllers. Finally, the data-oriented parameters are forwarded to regional SDN controllers, which in turn forward them to the local SDN controllers. Each local SDN controller forwards the received parameters to the members of its cluster.

\section{B. Automatic Data Collection}

Our proposed fog-assisted data collection scheme is proactive. This means that the data is collected and stored in fog stations even before user requests are generated. This ensures shorter response time (the time between requesting data and receiving it). The automatic data collection is carried out according to the data collection parameters already received from the global SDN controller. As illustrated in Algorithm 1, each sensed data item $(d)$ is collected only if it belongs to one of the categories defined by the global SDN controller. Once is established whether the data should be collected, the type of the data is checked to determine how quickly the data will be routed to the fog station.

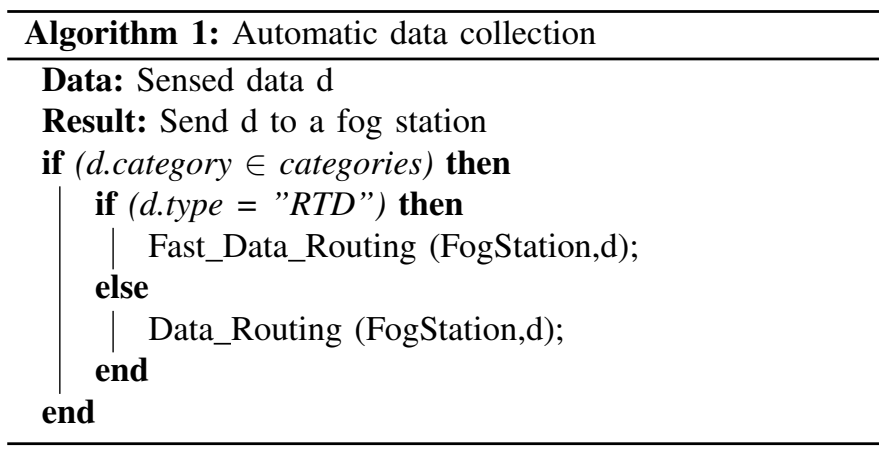

\section{Data request}

We distinguish two types of users: (i) system users: these users directly request data from the global SDN controller; and (ii) vehicular users: these users send their requests to local SDN controllers, specifying the category of the requested data and its corresponding geographic zone.

\section{Data collection parameter update}

- Importance factor: This parameter is periodically updated based on the requests of users. As depicted in Formula 1, this parameter is calculated by dividing the 
number of requests for a given category $\left(N b R_{i}\right)$ by the total number of requests.

$$
F I_{i}=\frac{N b R_{i}}{\sum_{1}^{n} R j}
$$

- Fog assignment: Due to the high mobility of vehicles, the assignment of fog stations should periodically be updated. To this end, the local SDN controller running at the $\mathrm{CH}$ level requests a fog station from the regional SDN controller (at the RSU), which checks for the nearest fog station to this $\mathrm{CH}$. If it exists, the regional SDN controller sends the ID of this fog station to the local SDN controller. Otherwise, it forwards the request to the global SDN controller, which, in turn looks for a fog station near the cluster. If a fog station is found, the global SDN controller forwards its ID to the local SDN controller. Else the global SDN controller deploys a fog station near to the cluster or creates a vehicular fog station. Once the newly created fog station is assigned to the cluster, the local SDN controller updates all its cluster members.

- Deployment of fog stations: The number of fog stations should be optimized to reduce the deployment cost for fog stations. Therefore, the global SDN controller periodically estimates the number of fog stations to be deployed according to vehicle density and the availability of the requested data as described in the next section $\mathrm{V}$.

- Data migration: Once our scheme has calculated the optimal number of fog stations to be deployed in the vehicular network, new fog stations are created or other stations may be removed. To prevent the loss of data, the collected data must be migrated from the old fog stations to the new fog stations.

\section{AnAlytical Estimation of Optimal Number of FOG STATIONS}

In this section, we provide a stochastic model to estimate the number of fog stations to be deployed in a given road area and as a function of time. We define the stochastic model as follows: Let the vehicle arrival distribution $V_{A}$ follow a Poisson process $P$ in a given road area. The inter-arrival time $t_{A}$ of $V_{A}$ has an exponential distribution with parameter $\lambda$. During its sojourn in the road area, a vehicle may collect data and send it to its corresponding fog station. For this reason, we define another random process $K$, independently of the arrival stochastic process $P$. As vehicles enter the road area, $K$ marks some of them with a positive probability $\rho(t) . \rho(t)$ models the probability that a vehicle collects data. Thus, vehicles that are not marked by $K$, will not send data to fog stations. In addition, $\rho(t)$ is a time-dependent probability because it depends on the traffic density in the road area during a given period. Indeed, the denser the traffic, the greater the number of vehicles that will send data to the fog stations. We also assume a random process $S$ that defines the time that will be spent by each vehicle inside the considered road area. This residency time can then be modeled by a random variable $(L)$ with a distribution function $F_{L}$. The vehicles' residency time is independent of the arrival process $P$ and the marking process $K$.

At $t=0$, we assume there is no vehicle inside the road area. Let $\{X(t) \mid \mathrm{t} \geq 0\}$ be the process that counts the number of vehicles which collect data during their residency in the road area. We are then interested in $E[X(t)]$ : the expected number of marked vehicles that reside in the road area at time $t$. According to the law of total probability, we have:

$\operatorname{Pr}[X(t)=k]=\sum_{n \geq 0} \operatorname{Pr}[X(t)=k \mid Y(t)=n] * \operatorname{Pr}[Y(t)=n]$.

Where $\operatorname{Pr}[X(t)=k \mid Y(t)=n]$ is the conditional probability that $X(t)=k$ given $n$ vehicles that enter the road area in the period $[0, t]$. Knowing that the vehicles' arrival follows a Poisson process we then have:

$\operatorname{Pr}[X(t)=k]=\sum_{n \geq 0}\left(\begin{array}{l}n \\ k\end{array}\right)[\gamma(t)]^{k}[1-\gamma(t)]^{n-k} \frac{[\lambda t]^{n}}{n !} \exp ^{-\lambda t}$.

$\gamma(\mathrm{t})$ is the probability that a given arriving vehicle is marked with a positive probability by $K$ and that it remains in this area at time $t$. Given that $n \geq 0$ vehicles have arrived in $[0, t]$, and their arrival times are uniformly distributed in $[0, t], \gamma(\mathrm{t})$ is given as follows [14]:

$$
\gamma(t)=\frac{1}{t} \int_{0}^{t} \rho(t-u)\left[1-F_{G}(u)\right] \mathrm{d} u
$$

Where $u$ is an uniform random variable in $[0, t]$. [14] demonstrated that:

$$
\operatorname{Pr}[X(t)=k]=\frac{[\Lambda(t)]^{k}}{k !} \exp ^{-\Lambda(t)}
$$

where $\{X(t) \mid t \geq 0\}$ is a Poisson process with parameter:

$$
\Lambda(t)=\lambda \int_{0}^{t} \rho(t-u)\left[1-F_{G}(u)\right] \mathrm{d} u .
$$

and with an expectation $E[X(t)]$ :

$$
E[X(t)]=\Lambda(t)=\lambda \int_{0}^{t} \rho(t-u)\left[1-F_{G}(u)\right] \mathrm{d} u .
$$

After estimating the number of vehicles collecting data during their sojourn period in the area, we can then predict the number of fog stations to be deployed in a given road area, which is denoted by $N_{f o g}(t)$ and given by the following formula:

$$
N_{f o g}(t)=\frac{E(X(t)) * Q}{C_{f o g}} .
$$

Where $C_{f o g}$ is the maximum storage capacity of a fog station, which is fixed by the global SDN controller. $N_{f o g}(t)$ denotes the maximum number of required fog stations for a given road area at instant $t ; Q$ is the maximum amount of data that could be collected by a vehicle. 


\section{Vi. Performance Evaluation}

\section{A. Estimation of the number of fog stations}

We make an analytical evaluation of our stochastic model for estimating the number of fog stations to be deployed. This evaluation is based mainly on the formula (2). We used real traffic measurements, which consist of 24 hours of traffic mobility for Luxembourg city [15]. We assume that the sojourn periods of vehicles inside the city are exponentially distributed following the distribution function $F_{L}=1-e^{-\mu t}$ with a mean equals to $30 \mathrm{~min}$. All vehicles are marked with a data collection probability at 0.5 during their residency in the city. Additionally, we set the maximum amount of data that a vehicle may collect to $10 \mathrm{MB}(Q)$ and the maximum capacity of a fog station to $100 \mathrm{MB}\left(\mathrm{C}_{f o g}\right)$. Figure 3 shows the hourly estimation of the number fog stations to be deployed using our proposed stochastic model. It is clear that the estimation of the number of fog stations accurately follows the volume of data available for collection. This volume depends mainly on the road traffic in the city, which naturally follows the daily routine of drivers. Indeed, a large volume of data is collected by vehicles in the morning and the evening. As a result, the number of fog stations allocated should be increased at these periods (37 and 39 fog stations need to be deployed at $9: 00$ and $19: 00$ respectively). This number decreases during offpeak hours. For instance, only ten fog stations are needed at $23: 00$.

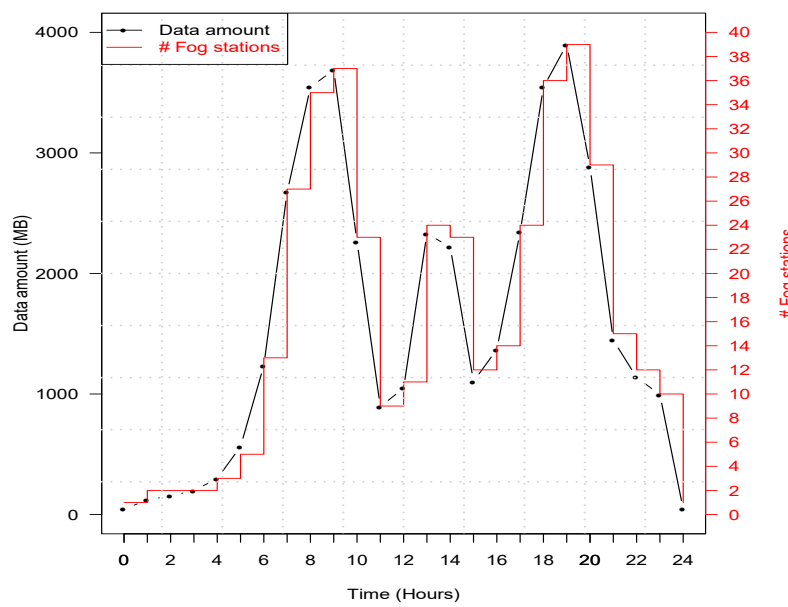

Fig. 3: Estimation of the number of fog stations in Luxembourg City scenario

\section{B. Performances of the fog-assisted data collection scheme}

We also carried out extensive simulations to evaluate the performance of our proposed fog-assisted data collection scheme. These simulations were conducted using Veins Simulation Framework [16]. Table I summarizes the parameters considered in our simulations.

Our focus was on evaluating the impact of SDN on our proposed data collection scheme. For this reason, we considered the case of a freeway. We simulated a two-lane straight road section. The mobility of vehicles was generated using SUMO [17]. As shown in Table I, we considered, 10 deployed
TABLE I: Simulation Parameters

\begin{tabular}{ll}
\hline Parameter & Value \\
\hline Simulation duration & $120 \mathrm{~s}$ \\
Transmission Range & $500 \mathrm{~m}$ \\
The size of the cluster & $\{10,20,30,40,50\}$ \\
Number of RSUs & 10 \\
$\mathrm{C}_{f o g}$ & $100 \mathrm{MB}$ \\
$\mathrm{Q}$ & $10 \mathrm{MB}$ \\
\hline
\end{tabular}

RSUs along the freeway. The fog stations are deployed in these RSUs. We also considered one cluster of vehicles whose size was varied from 10 to 50 vehicles. Every five seconds, vehicles send the data they collect to their assigned fog station. In our simulation, we adopted an intra-RSU routing protocol i.e. each collected data item is routed through RSUs until it reaches the assigned fog station. We compared our SDN-based scheme with a classical scheme that statically and randomly assigns a fog station to the cluster. Two metrics of comparison were used: (i) the latency, which is the time taken by the collected data to reach the fog station, and (ii) the number of hops that are needed to reach the fog station. This second metric is considered because a packet that traverses a large number of hops has more chances to be retransmitted, which results in high overhead and consequently more congestion in networks. We repeated the simulation several times with different random seeds and calculated the average value with a $95 \%$ confidence interval.

In Figures $4 \mathrm{a}$ and $4 \mathrm{~b}$, we compare our SDN-based scheme with the classical scheme in terms of the average latency and the number of hops respectively. The SDN-based scheme drastically reduces the latency and the number of hops. The reason for this is that our scheme assigns the nearest fog station to cluster in each update process (see Figure 4b). However, in the classical scheme, the fog station is randomly, chosen without considering the mobility of the cluster.

In Figure 4c, we evaluate the delay needed to route both delay tolerant data (DTD) and real-time data (RTD) under different sizes of the cluster. As we can see, the RTD are routed faster than DTD. We can also see that the latency for routing DTD increases with the size of the cluster. However, the latency of RTD generally remains stable, whatever the cluster size is. This can be explained by noting that our scheme takes the data type into the account and prioritizes RTD over DTD.

In Figure 5, we evaluate the resiliency of our scheme against the failure of the assigned fog station, since fog nodes are typically equipped with capacity-limited batteries. We compare our proposed scheme with the classical. Two metrics are used: (i) the recovery time, and (ii) the data loss. As we can see, the recovery time of our scheme is much less than the that of classical scheme, because once the failure is detected, the regional SDN controller starts searching for a new fog station to host the collected data and assigns the new fog station to the cluster. The loss of data in our scheme is also much less than the classical scheme, since the data will immediately be forwarded to the new fog station. 


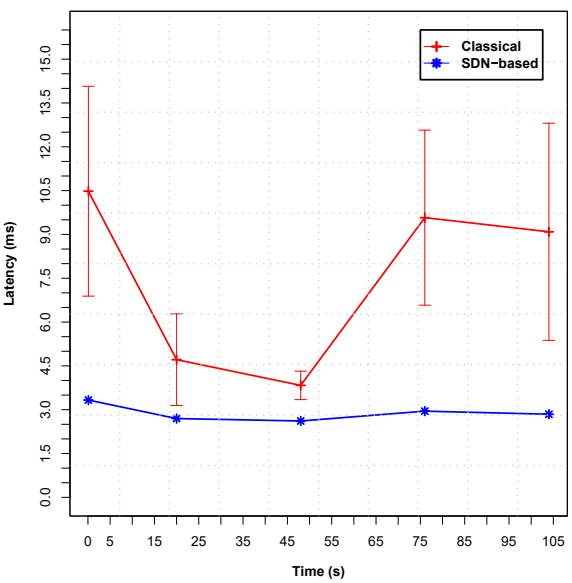

(a) Average of latency.

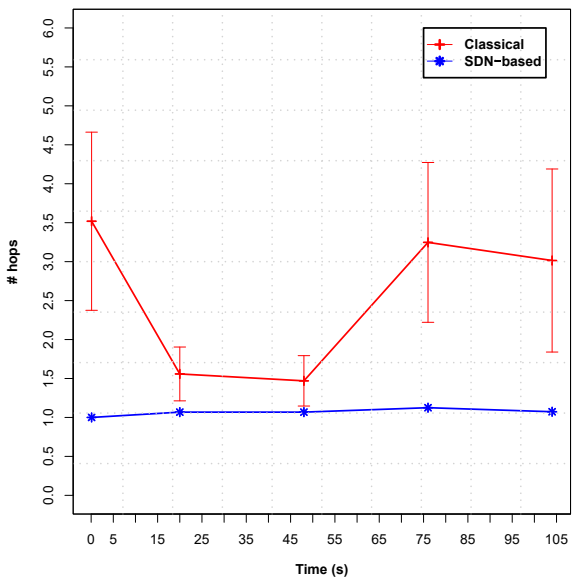

(b) Average number of hops

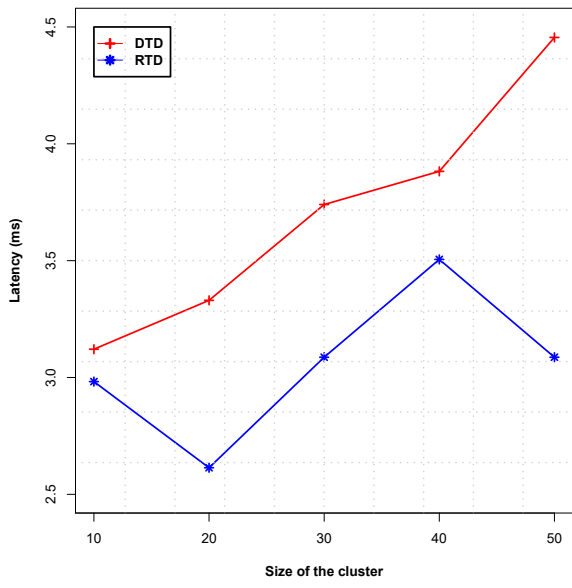

(c) Latency of routing (cluster size $=50$ )

Fig. 4: Latency and the number of hops
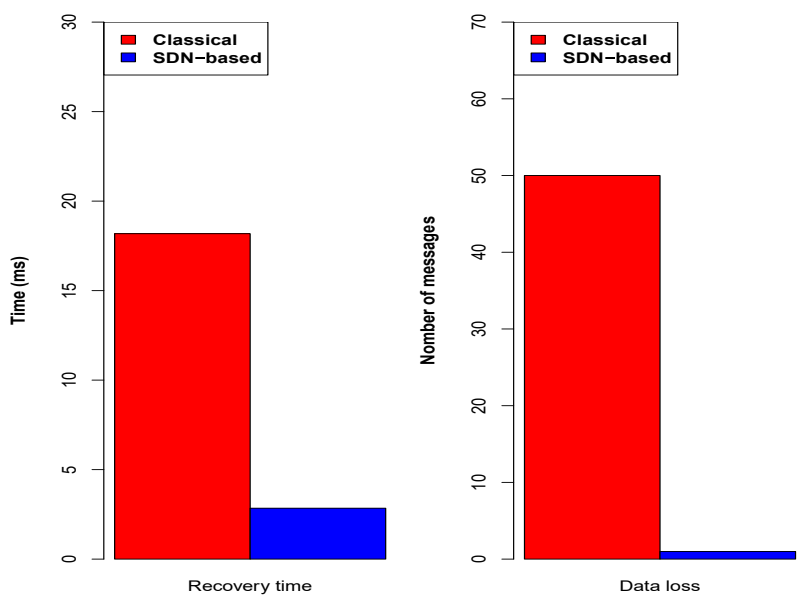

Fig. 5: Recovery time \& data loss after a failure

\section{CONCLUSION}

In this paper, we propose a novel fog-assisted data collection scheme in SDN-enabled vehicular networks. This scheme is fully programmable, self-configurable, and context-aware leveraging an accurate stochastic model to estimate the number of fog stations to be deployed according to the traffic density and the availability of data. We have validated this model using realistic road traffic measurements. Finally, the simulations we conducted have shown the merit of our scheme compared to a classic data collection scheme in terms of delay, recovery time and data loss.

\section{ACKNOWLEDGMENT}

This work was supported by the H2020 5G-DRIVE project (ID: 814956).

\section{REFERENCES}

[1] K. Golestan, R. Soua, F. Karray, and M. S. Kamel, "Situation awareness within the context of connected cars: A comprehensive review and recent trends," Information Fusion, vol. 29, pp. 68 - 83, 2016.

[2] F. Dressler, P. Handle, and C. Sommer, "Towards a vehicular cloud using parked vehicles as a temporary network and storage infrastructure," in
Proceedings of the 2014 ACM international workshop on Wireless and mobile technologies for smart cities. ACM, 2014, pp. 11-18.

[3] M. Chaqfeh, N. Mohamed, I. Jawhar, and J. Wu, "Vehicular cloud data collection for intelligent transportation systems," in 2016 3rd Smart Cloud Networks \& Systems. IEEE, 2016, pp. 1-6.

[4] J. C. Nobre, A. M. de Souza, D. Rosario, C. Both, L. A. Villas, E. Cerqueira, T. Braun, and M. Gerla, "Vehicular software-defined networking and fog computing: Integration and design principles," $\mathrm{Ad}$ Hoc Networks, vol. 82, pp. 172-181, 2019.

[5] C. Huang, R. Lu, and K.-K. R. Choo, "Vehicular fog computing: architecture, use case, and security and forensic challenges," IEEE Communications Magazine, vol. 55, no. 11, pp. 105-111, 2017.

[6] R. Soua, I. Turcanu, F. Adamsky, D. Führer, and T. Engel, "Multi-access edge computing for vehicular networks: A position paper," in 2018 IEEE Globecom Workshops (GC Wkshps). IEEE, 2018, pp. 1-6.

[7] Y. Lai, L. Zhang, T. Wang, F. Yang, and Y. Xu, "Data gathering framework based on fog computing paradigm in VANETs," vol. 10612, pp. 227-236, 2017.

[8] Y. Lai, F. Yang, J. Su, Q. Zhou, T. Wang, L. Zhang, and Y. Xu, "Fogbased two-phase event monitoring and data gathering in vehicular sensor networks," Sensors, vol. 18, 2017.

[9] B. Pourghebleh and N. Jafari Navimipour, "Towards efficient data collection mechanisms in the vehicular ad hoc networks," International Journal of Communication Systems, vol. 32, no. 5, p. e3893, 2019.

[10] I. Turcanu, P. Salvo, A. Baiocchi, and F. Cuomo, "An integrated VANETbased data dissemination and collection protocol for complex urban scenarios," Ad Hoc Networks, vol. 52, pp. 28-38, 122016.

[11] R. F. Drira, Ahn, "Development and testing of a 3G/LTE adaptive data collection system in vehicular networks," IEEE Transactions on Intelligent Transportation Systems, vol. 17, no. 1, p. 240-249, 2016.

[12] D. T. Z. Jiao, Ding, "Predictive big data collection in vehicular networks: A software defined networking based approach," 2016 IEEE Global Communications Conference (GLOBECOM), 2016.

[13] X. Ji, H. Yu, G. Fan, and W. Fu, "SDGR: An SDN-based geographic routing protocol for VANET," in 2016 IEEE International Conference on Internet of Things (iThings) and IEEE Green Computing and Communications (GreenCom) and IEEE Cyber, Physical and Social Computing (CPSCom) and IEEE Smart Data. IEEE, 2016, pp. 276-281.

[14] G. Yan, S. Olariu, J. Wang, and S. Arif, "Towards providing scalable and robust privacy in vehicular networks," IEEE Transactions on Parallel and Distributed Systems, vol. 25, no. 7, pp. 1896-1906, 2013.

[15] L. Codeca, R. Frank, and T. Engel, "Luxembourg SUMO traffic (LUST) scenario: 24 hours of mobility for vehicular networking research," in 2015 IEEE Vehicular Networking Conference (VNC). IEEE, 2015.

[16] C. Sommer, R. German, and F. Dressler, "Bidirectionally coupled network and road traffic simulation for improved IVC analysis," IEEE Transactions on Mobile Computing, vol. 10, no. 1, pp. 3-15, Jan 2011.

[17] SUMO, "Simulation of urban mobility," http://sumo.sourceforge.net/, 2019. 